Fecha de recepción: abril 2019 Fecha de aceptación: octubre 2019 Versión final: marzo 2020

\section{Educar para no competir. La guerra de las naciones: nuevo escenario multipolar e innovación social como alternativa de adaptación}

Mercedes Buey Fernández *

\begin{abstract}
Resumen: En un mundo cambiante y globalizado, que pide a gritos innovación, identidad y significado, pareciera ser que aquellos que esperan que el mundo se ajuste a sus cálculos y predicciones están destinados a ser frustrados. Históricamente los cambios han dado miedo porque lo desconocido asusta. Sin embargo, la vida hoy se muestra intrínsecamente cambiante, conmovedora, decepcionante y sorprendentemente positiva. En este sentido, vivir una vida de expectativas inflexibles es una receta al desastre (Asma, 2017). En definitiva, las reglas y estructuras no son creaciones divinas, ¿qué se hace cuando caen las estructuras donde se vivió toda una vida?

El mundo se encuentra hoy sumido en tiempos de cambios drásticos. Las potencias se debilitan, las masas se dispersan y la tierra llora. Pero la humanidad ya no teme al cambio, busca el cambio; el seguidor de reglas se frustra y el improvisador se adapta.
\end{abstract}

Palabras clave: sociología - globalización - cultura - debate - diseño - innovación - actualidad.

[Resúmenes en inglés y portugués en las páginas 89-90]

${ }^{(*)}$ Diseñadora Industrial (UP). Profesora de la Universidad de Palermo en el Área de Diseño de Objetos y Productos de la Facultad de Diseño y Comunicación desde 2012. A lo largo de su trayectoria se desempeñó como diseñadora senior y semi senior en diferentes empresas. Desde 2012 es socia gerente, diseñadora y fundadora de Estudio De Raíz S.R.L. y Yerba Mate Mathienzo, fue docente evaluadora de Proyectos de Graduación; es especialista en Gestión de Diseño (UBA) y asistente externa en el área de estudios ergonómicos y capacitaciones de diferentes empresas.

\title{
Introducción
}

En un mundo cambiante y globalizado, que pide a gritos innovación, identidad y significado, pareciera ser que aquellos que esperan que ese mundo se ajuste a sus cálculos y predicciones están destinados al desastre total. A lo largo de los años, la respuesta a las cuestiones de agenda humana parecía permanecer invariable. Los sucesos eran acciones encadenadas de la naturaleza a las que la humanidad se enfrentaba sin cuestionar causales, o al menos esa era la concepción reinante. 
Sin embargo, la humanidad hoy descubre cosas asombrosas.

El escenario económico, político, geográfico y social está cambiando y esto impacta e importa a todos por igual. Desde principios del siglo XXI los cambios se presentan de manera vertiginosa, de toda índole y las cuestiones de agenda ya no son hechos incontrolables de la naturaleza, para pasar a ser cuestiones manejables, la incidencia de diferentes calamidades ha disminuido y cuando alguna parece salirse de control, enseguida alguna fuerza organizativa logra dominarla. Las estructuras hasta ahora conocidas se desmoronan, los límites y las fronteras se desdibujan, la tierra llora y pide a gritos un cambio actitudinal. Las políticas proteccionistas, la cuestión migratoria y las guerras modernas -luchas indiscriminadas por el poder, que esconden motivaciones económicas y/o geoestratégicas, detrás de una falsa búsqueda de la liberación y patriotismo- hacen que la vida se muestre hoy intrínsecamente cambiante, conmovedora, decepcionante y sorprendentemente positiva (Asma, 2017).

En líneas muy generales, el Brexit ha debilitado a la Unión Europea, el terrorismo islámico es una amenaza para occidente y Rusia está emergiendo como nueva potencia. Los analistas y expertos en geopolítica afirman que en los próximos años China será la primera superpotencia, la economía de Brasil pasará del noveno al cuarto lugar en el plano internacional y países como México, Vietnam o Indonesia podrían desarrollarse significativamente (Javier Navarro, 2017).

Sin embargo, ese crecimiento está supeditado, en gran medida, a un crecimiento económico y tecnológico que nos proporciona lo necesario para la supervivencia humana-comida y medicinas por ejemplo-, pero desestabiliza el equilibrio geo-ecológico del planeta de muchísimas maneras. Los avances en la brecha tecnológica han afectado y han conseguido cambiar el ecosistema global de formas radicales y sin precedentes.

Como actores principales de este mundo en clara transición el interrogante más fuerte sea seguramente ¿cómo navegar en estas aguas turbulentas?, ¿qué exigirá la atención en este nuevo marco coyuntural?, y, más precisamente: ¿cómo van a reflexionar y adaptarse los profesionales, a nuevos modos de comunicación y diseño, de manera tal que el público sienta empatía con el trabajo realizado y se llegue a una identificación y entendimiento pleno?

Autores como Harari (2017) se animan a delimitar la nueva agenda humana y en su análisis afirman que hace años el saber humano aumentaba despacio, de modo que la economía y la política cambiaban también a un ritmo pausado. Hoy, el crecimiento se da a una velocidad de "vértigo", y lo que en la teoría significaría que deberíamos entender al mundo cada vez mejor, en la práctica el presente se muestra demasiado distinto al pasado, y la capacidad de dar sentido a ese presente o de pronosticar futuro, como él sostiene, o estudiar el pasado, sólo encuentra justificación en el afán de "no repetirlo, de liberación". En definitiva, "el estudio de la historia no nos dirá qué elegir, pero a al menos no dará más opciones" (p. 74).

Por esto, todas las predicciones esbozadas en este ensayo se hacen eco de su visión y no pretenden ser otra cosa más que un dedicado intento de analizar sucintamente los dilemas actuales e invitación a cambiar el futuro, no tanto como una profecía, pero como una forma de analizar las opciones (Harari, 2017, p. 80). Hacer un análisis de contexto y predecir que el mundo está en transición y que esa transición es el resultado de la búsqueda de un 
fin común, resulta algo obvio, pero sólo se pueden pensar alternativas una vez que uno se enfrenta directamente a esos dilemas actuales.

El objetivo entonces, es lograr mejores decisiones, no mejores predicciones. Lo que las transforma a veces en pronósticos acertados no es que ellos como especialistas tengan la capacidad de prever el futuro, sino que lo que los hace verdaderamente buenos es que se ocupa de mirar el presente con mayor detenimiento (Evans, 2007).

¿Qué utilidad tendría si no, el nuevo conocimiento, más que el de llevar a uno a desarrollar comportamientos nuevos? No se pretende con esto hacer futurología, ni se pretende aquí vaticinar pronósticos, sencillamente porque como se expuso más arriba: el presente es muy distinto al pasado y los cambios se suceden a ritmos vertiginosos y de manera inesperada. Históricamente los cambios han dado miedo porque lo desconocido asusta. Sin embargo, vivir el cambio más que esperar para analizarlos, pareciera ser el modo de obrar que rige. Hoy ya no hay tiempo para ver cómo se acomodarán los límites y jugadores del nuevo mapa geopolítico. La humanidad no tiene tiempo de esperar para revisar y comenzar a reescribir la historia. La escribe mientras los hechos se suceden, incluso sin darse cuenta del peligro que eso podría conllevar para con la humanidad misma.

En este sentido, un proyecto central será proteger a la humanidad y al planeta en su conjunto de peligros inherentes a su propio poder. Hasta ahora aumentar el poder humano se basaba principalmente en mejorar las herramientas externas, aumentar la red de conexiones, $y$ afianzar las interrelaciones pero, en un futuro, las mejoras en las condiciones internas, el aumento del conocimiento y la descentralización serán la base de todo poder. (Harari, 2017). Porque si hasta ahora la humanidad se definió por lo externo, global y lo universal, de ahora en más se va a definir por una búsqueda de ética ulterior, unido a conceptos tales como tejido social, territorio, ciudadanía, diversidad cultural, paz y hasta el concepto de resiliencia. Los factores clave que servirán de argumentación para el debate serán la desigualdad y la migración; y la meta será lograr un impacto inclusivo.

\section{Nuevo mapa geopolítico: ¿Fin de la globalización?}

Las diferentes investigaciones muestran que el mundo está mucho menos concentrado que en los años ochenta, y que ha crecido constantemente desde las últimas dos décadas, impulsado principalmente por el crecimiento de China como se mencionaba anteriormente. Cuando Estados Unidos y la URSS lideraban el escenario político internacional, el mundo se dividía en dos bloques claramente diferenciados. Había dos ideologías antagónicas, el sistema democrático de los países occidentales liderados por Estados Unidos frente al modelo comunista de partido único que impuso la URSS en toda Europa oriental.

Desde un punto de vista económico, tomando en análisis preexistentes, Estados Unidos y sus países aliados impulsaron un modelo capitalista basado en el libre mercado y el bloque soviético mantuvo una economía planificada y basada en la intervención estatal. Con la desintegración de la URSS, parecía inicialmente que el mundo tendría una única superpotencia: Estados Unidos. Pero, este país, líder indiscutido durante décadas, en cambio, ha ido perdiendo una parte significativa de su hegemonía en el orden internacional. Lo que valida una sensación térmica de urgencia (Javier Navarro, 2017). 
Actualmente, el miedo está ganándole la pulseada a la población europea frente a los hechos de terrorismo. Los atentados se suceden, y cada vez más en países donde hasta hace algunas décadas era impensado, como en Francia, país que a su vez concentra un gran grupo de refugiados musulmanes, característica que alimenta la división étnica interna y el miedo de represalias de tipo religiosas. A partir de eso, todo se sucede como efecto dominó, el miedo hace que las poblaciones no estén abiertas a recibir refugiados, que quieran fronteras más fuertes y cerradas, y que exijan que primero se garanticen sus derechos, frente a los de los refugiados. Esto hace que los diferentes partidos políticos de extrema derecha estén ganando cada vez más terreno en Europa, y se aliente el cierre de fronteras. En paralelo, no se puede obviar la situación bélica en Siria, con la toma de tierras por parte del ISIS y la consecuente migración de sirios escapando del país.

Indefectiblemente, esta sensación de inseguridad, y realidad, se traslada a Estados Unidos, donde el escenario se muestra aún más complejo. El país norteamericano hace décadas convive con el terrorismo y tiene un continuo flujo de inmigrantes de todo el mundo que ingresa al país - de manera legal e ilegal- en busca de trabajo y un futuro mejor. Por su parte, la población estadounidense valida la sensación de que el país perdió sus aires de grandeza, y comienza a observar que la facilidad con la que antes se accedía a los empleos se perdió y que alcanzar famoso sueño americano que siempre les prometieron, es cada vez más difícil. Es un hecho que los inmigrantes son cada vez más, que las desigualdades se profundizan, y que la crisis financiera de 2008 no hizo más que acentuar la situación, teniendo como consecuencia una profunda crisis de liquidez interna, y derramándose luego las consecuencias en el plano económico internacional.

Comienzan a tener preponderancia en este nuevo escenario geopolítico figuras como la de Donald Trump y su discurso político totalmente acomodaticio, ventajero y oportuno, en un contexto en el que sus rivales no supieron aprovechar. Trump tocó fibras y apeló a la emoción de la población, culpando a los inmigrantes de todo mal, prometiendo muros y asegurando que América -en clara referencia únicamente a Estados Unidos- sería grande de nuevo. -Slogan acuñado por Reagan en 1980 y utilizado por Trump en la campaña presidencial 2016-.

El panorama sudamericano, en este fin de siglo, no podría ser peor. Se están terminando los populismos contemporáneos y aquellos gobiernos que no comulgaban con la propuesta del bloque dominante, priorizaban el crecimiento y la distribución de ingresos, sin preocupaciones suficientes por los riesgos consecuentes como inflación, déficit financiero, devaluaciones, etc., y tenían leyes benignas para la inmigración -con los xenófobos y racistas de la derecha extrema- están mutando hacia una creencia mucho más antagónica y una situación de crisis profunda que da por supuesto el inicio de transformaciones políticas y económicas de fondo.

La afirmación sería pues, que el cambio que se espera ver si el mundo sigue este camino, será el del desarrollo de nuevas instituciones, sumado a una nueva situación migratoria regional y urbana por sobre la transfronteriza, y el surgimiento de nuevos centros financieros de índole local. A nivel corporativo, el cambio más importante podría llegar a darse con el aumento de las compañías regionales, y sobre todo, al aplastamiento económico de estas para con las multinacionales existentes. Se proyectan mejoras en el desarrollo humano, sobre todo en relación a las desigualdades existentes, que conduzcan a una mayor 
estabilidad y el desarrollo de economías locales más ricas y fuertes gracias a la continuidad de la tendencia de los consumidores de mercados emergentes. Esta tendencia alimenta el proteccionismo y la sustitución de importaciones, y en consecuencia, las políticas comerciales orientadas hacia el interior ganan cada vez más terreno, a medida que las economías buscan impulsar el empleo y la actividad económica dentro de las fronteras (Flores, 2017). Esto efectivamente pone en jaque el concepto de globalización, modernización y educación, como se conocía hasta ahora, para pasar a un mundo multipolar, siendo ese el escenario más realista. Y, en este sentido, vivir una vida de expectativas inflexibles es una receta al desastre (Asma, 2017).

\section{La concepción de un mundo multipolar}

En primera instancia cabe aclarar que aquí se entenderá a la globalización desde el aspecto económico y se la concebirá como "proceso por el cual dos o más estados deciden estrechar sus relaciones económicas, creando mecanismos para la integración gradual de sus economías"(Dabbah, 2015). Esto lo que busca es la supresión de muchas de las barreras arancelarias, para favorecer la libre circulación de capitales -financieros, comerciales y productivos- con el objetivo de alcanzar la libre movilidad de ese capital y la creación de un solo mercado mundial. Este tipo de integración ha cambiado hasta el momento el rumbo de la historia en diferentes ámbitos, ampliando el espacio económico entre países que tenían mercados separados, constituyendo un solo mercado de dimensiones mayores, e incluso disminuyendo conflictos internacionales a raíz de la dependencia recíproca que se generaba a partir del estímulo comercial (Villamizar, 2000).

Actualmente, estas relaciones de interdependencia que se perpetraron con la fantasía de que la globalización hacía accesible el mundo al mundo en general, están agrietándose. Durante décadas se enmascararon las desigualdades y asimetrías provocadas por la falta de integración económica real (Arroyo Madera et al., 2015). Hace tiempo se tiene la evidencia de que la globalización y homogeneización sólo corren paralelos en las fantasías utópicas o en el imaginario fantástico de aquellos que tienen menos que perder y mucho para ganar. Asimismo, como también se analizó párrafos más arriba, desde el punto de vista geográfico no existen los países centrales ni periféricos. El concepto es exclusivamente de naturaleza política, y hoy se está redibujando. Los politólogos actuales comienzan a esbozar sus definiciones de multipolaridad, y la proponen como alternativa de adaptación al cambio. La más utilizada es la que asegura que habrá un desarrollo económico político y social dividido por regiones, en detrimento de la falsa integración e interdependencia mundial provocada por la globalización (Flores, 2017).

En este contexto, la teoría de un mundo multipolar representa una dirección única que ya no se vale de calificaciones reduccionistas como progreso versus conservadurismo, o viejo versus nuevo. La visión globalista de muchos pretende analizar el fenómeno como un proceso lineal en el que del subdesarrollo se pasa al desarrollo, y eso se mide de manera cíclica. Aunque es poco probable que la globalización se detenga por completo, quienes tienen la visión de un mundo multipolar, sostienen que el concepto de irreversibilidad de la globalización no puede probarse del todo cierto. Es verdad que el mundo universal y 
globalizado al que muchos se han acostumbrado ha tomado otras formas; pero el retorno a cero o el viraje a un estado previo total, es poco probable, puesto que la conectividad y la inmediatez de la información y la internet hace rato han transformado el modo en el que la humanidad se interrelaciona, personal y económicamente.

En este caso, se cree que es posible dejar de lado el "dogmatismo de un proceso lineal o monótono", para pasar a la interpretación del paradigma multipolar como un "proyecto de organización de orden mundial bajo principios y elementos absolutamente nuevos", permitiéndose una "revisión importante de los axiomas ideológicos, filosóficos y sociológicos sobre los que descansa la modernidad" (Dugin, 2018).

La diferencia fundamental entre la visión globalista, y la multipolaridad es que mientras la primera defiende a ultranza intereses comerciales que no son otra cosa más que el enmascaramiento de acuerdos entre unas pocas partes interesadas en obtener un rédito inmediato, sin medir las consecuencias a escala mundial; la segunda detecta en la sociedad una tensión creativa y un esfuerzo por construir una sociedad más feliz, justa y armoniosa. Observan al futuro como diferente y desigual y hasta conservador dentro de la amplia paleta de opciones de sociedades que experimentan la influencia de distintas matrices de identificación. ¿La consecuencia? Una floreciente complejidad del mundo, donde múltiples actores individuales y colectivos se sumergen en un diálogo para transformar re-encontrar su identidad. Se plantean nuevos escenarios, y un nuevo paradigma basado en diseños éticos por sobre los estéticos. Y lo que es más importante, que esa ética emergente se muestra preocupada por el medio ambiente y el desarrollo local. El compromiso social demuestra ser hoy el $100 \%$ del proyecto, y estar a su vez compuesto por un $90 \%$ de empatía, compromiso y sensibilidad; y un 10\% de diseño -entendido este como la acción de proyectar objetos útiles- (Cambariere, 2017).

El dilema puntualmente entonces es, ¿cómo logran los diseñadores en -este caso- acomodarse en este nuevo escenario coyuntural? El colectivo se ve hoy obligado a desarrollar su costado más sensible y mostrarse cada vez más receptivos a determinados asuntos o problemas y proclives a ponerles solución.

En esta nueva era del diseño, el diseño artesanal resulta ser la estrella, y esa energía creativa (Berman, 1992) que en algún momento se expandió sin límites por doquier, hoy se dosifica. El mundo se encoge y lo que alguna vez se pensó como federativo, hoy se reconoce con signos de dominación y competencia más que cooperación (Santos, 1993).

\section{Replanteo artesanal}

Durante años los referentes de la disciplina incorporaron fórmulas que les eran ajenas y no sólo soslayaron voces propias, sino que, impusieron un paradigma insostenible y muy limitante. Lo bueno es que ese paradigma se está resquebrajando y se plantean nuevos escenarios para el diseño, donde este entra en contacto directo con los seres humanos y el desarrollo local es inevitable (Cambariere, 2017).

A diferencia de años anteriores, donde la transición que se vaticinaba era de un mundo globalizado hacia uno glocalizado con la intención de entender las maneras globales actuando localmente para producir nuevas culturas (Buey Fernández, 2017), la tendencia 
actual pretende el rescate de técnicas, materiales y un cuidado del medio ambiente que básicamente sirvan de ejemplo al mundo. Ya no se pretende llegar al mundo con diseños que gusten al mundo, sino que se pretende llegar al mundo con lo propio.

La incorporación de la historia a la mirada moderna es el factor clave de evolución. La humanidad entiende ahora que el binomio estrella tiene que ser el diseño de la mano de lo social para el desarrollo de nuevas y mejores economías.

En este contexto de cambios permanentes, controlar y reducir la incertidumbre del entorno se ha vuelto tarea casi imposible, pero, se trata de entender que esta nueva oportunidad exige profesionales con una mirada mucho más seria y responsable para con el contexto que los recibirá. Profesionales capaces de modificar positivamente el entorno en el que se mueven, y capaces de reconocer los continuos cambios, necesidades, expresiones, requerimientos y tendencias del contexto actual. Y capaces de analizar en forma crítica los requerimientos y valorarlos de manera integral, detectando las implicaciones estéticocomunicativas, técnico-productivas; así como éticas, económicas, políticas y ambientales de sus propuestas, para proyectar no nuevas culturas, sino, nuevos productos y con ellos, nuevos lenguajes comunicativos.

Las nuevas oportunidades de interacción conducen, de un modo u otro, al proceso clave de detección de oportunidades de diseño, y no necesariamente necesidades de diseño. La mirada está enfocada en el por qué se hacen las cosas más que en la cosa en sí misma; y los esfuerzos por dotar de significado nuevamente al término diseño, son innumerables.

El diseño fue, es y será trascendental para la vida de las personas y el desarrollo humano en general. El buen diseño puede salvar vidas. El diseño media casi todas las acciones cotidianas de una persona. Casi todas las acciones que se hacen en el día a día están intermediadas por una interfaz de diseño, sea esta concebida o no por un diseñador. Desde una cuchara, hasta un avión, pasando por instrumental médico, todo, todo, está mediado por el diseño. Sin embargo, lamentablemente a veces las personas, incluso los mismos diseñadores, ligan al diseño a su aspecto más reduccionista de moda u objeto práctico-funcional únicamente, y mal cargan de significado al término. Pero ¿qué es el diseño en realidad?

El diseño tiene que ver con el proyectar un mundo mejor y no existe de otra forma más que concibiéndolo en relación a las personas. La mirada estratégica hoy condena en cierta medida a la mirada del primer mundo como único referente, para volver a reconocer en cada uno de los agentes partícipes de este mundo en transición, la revalorización del mínimo recurso que es el máximo; de la creatividad, la imaginación, el reciclar lo reciclado la búsqueda de recursos constantes. Sentirse parte de la naturaleza y trascender lugares comunes que hoy tiene el diseño y concentrarse en objetos menos analizados, para recobrar la esencia de las definiciones y lo artesanal de las vinculaciones. Esta nueva era de transición aspira a ser un nuevo espacio propicio para el diálogo, derribando prejuicios y echando luz sobre la pluralidad de creaciones de los diferentes pueblos.

Lejos de un perfil nostálgico, lo interesante del sentimiento actual es mostrar cómo las diferentes culturas se puedan encontrar, contraponer y dialogar, para transpirar una contemporaneidad tal que reinvente las lecciones del pasado. De transparentar las decisiones, de que todos formen parte de un saber popular que debe ser visto como fuerza vital para el desarrollo en este nuevo esquema geopolítico y nuevos mercados de los que se hablaba anteriormente. 
"Ni la modernización exige abolir las tradiciones, ni el destino fatal de los grupos tradicionales es quedar fuera de la modernización", explicaba el argentino Néstor Canclini (2010) entrevistado en marco de la inauguración de la muestra brasilera Puras Misturas inaugurada el 11 de abril de 2010 y que bien sirve como muestra clara de esta transición de la que tanto se viene hablando.

Dicha muestra creía vital la creación de un espacio de legitimación de diferentes prácticas culturales y sobre todo, de legitimar a aquellos productores de cultura y significados plurales, por encima del mero mote de consumidores. El aspiracional de ese tipo de exhibiciones es, gracias a la inclusión de temas, perspectivas, lenguajes, visiones, público, culturas, etc., servir como espejo para que el conjunto de ciudadanos de la región, en este caso Brasil, se reconozcan y sean reconocidos, tendiendo puentes entre la periferia y el centro, revelando, canalizando e irradiando el poder transformador de la creación popular (Borges, 2010).

\section{Innovación genuina}

Los diseñadores interactúan con las fuerzas de la modernidad de tal modo que los nuevos diálogos entre diseñadores, artesanos, autores y pensadores de la materia, tienen como objetivo primero el desarrollo de las poblaciones que quedaron desplazadas. Los nuevos escenarios se plantean propicios para la innovación estratégica sin dejar de lado la ética ni lo social.

Lo que para muchos estalló en la Argentina en 2001, para diseñadores como Beatriz Galán, se comenzó a vislumbrar en los años '90 y terminó de verificarse en los últimos años en el ámbito del diseño nacional e internacional donde hoy hay que explorar nuevos escenarios y nuevas formas de actuación, y donde el diseñador está obligado a asumir nuevos roles lejos de la innovación comprendida como un efecto meramente imitativo de lo que se ve novedoso en países centrales. En la región se comprobó que existen otros móviles, sobre todos los éticos, que tienen que ver pura y exclusivamente con el interés en el arraigo territorial, validando el esquema multipolar (2006).

Son esas condiciones coyunturales y circunstancias dadas del contexto las que generan los elementos y plantean los escenarios para alcanzar la tan deseada innovación genuina y estratégica. Es necesario entender que la innovación genuina de la que hablan los expertos, sólo será posible si se comprende que las problemáticas que ingresan en los territorios son, en la mayoría de los casos, problemas construidos y poco tienen que ver con los problemas locales, por eso, equivocadamente se rechaza el problema, cuando se debiera rechazar el marco teórico (Galán, 2006).

Para Eduardo Naranjo (2006), el que legitima es aquél que viene a buscar en el otro lo que el otro no tiene. Aquellos que van en busca de nuevos recursos y saberes, para apropiarse de esos y evitar migraciones indeseadas. El enfoque actual, en este sentido, es la interacción y la transferencia en la acción. El diseño no es un nuevo objeto, es un catalizador, un mediador, cómo se define antes en este escrito, para el diseño de un mundo nuevo y mejor. En el marco de las jornadas Diseño para el Desarrollo Local organizadas en la UBA en el año 2006, el diseñador dio cuenta de su postura acerca de esta visión de legitimiza- 
ción y de una experiencia en particular: Laboratorios de Paz. Una iniciativa especialmente ideada para las zonas de conflicto, las zonas rojas, que pretende de la mano de la cultura -fortaleciendo la artesanía, la música y el teatro- ser otra vía para reducir el impacto de la violencia. Estas iniciativas, son otra muestra de fortalecimiento de los actores involucrados para ordenar recursos con criterios de eficacia social; lograr comunidades mejores posicionadas y profesionales competentes para accionar en escenarios complejos, quebrar estereotipos y, lo más importante, volcar su atención a la realidad local en pos de descubrir su riqueza sin imitación (Naranjo, 2006).

\section{Educar para no competir}

La educación afronta hoy un desafío muy grande. En el marco de World Economic Forum que se realiza todos los años, también conocido como el Foro de Davos, Jack Ma (2018) afirmaba que para el año 2030 las máquinas reemplazarán 800 millones de puestos de trabajo ocupados por humanos. Por esto, si no cambia la forma en la que se enseña y sobre todo, lo que se enseña, en 30 años la humanidad estará en problemas.

Por consiguiente, frente a este panorama desalentador en la teoría, pero altamente positivo en la práctica, ¿cómo se siembran las semillas de un mundo posible?

La respuesta está en la educación. Se suele decir que educando bien a un niño/a hoy, ya nadie tendrá que preocuparse por el calentamiento climático el día de mañana. Y si bien la cuestión no es tan directamente proporcional y lineal, el concepto se logra comprender de buena manera.

Los modos actuales se basan en la enseñanza de conocimiento, en el desarrollo de habilidades duras y responde a preceptos algo arcaicos. Esos modelos basados en el conocimiento no sirven hoy para enseñar a las generaciones futuras a competir con las máquinas, ellas ya son más inteligentes.

Aquello a lo que te resistes, persiste, decía Carl Jung (s/f) en varios de sus escritos. Y no podría definir de mejor manera una actitud altamente enraizada en la mayoría de los seres humanos: la de aferrarse a aquello que es conocido y a las ideas e imágenes preconcebidas, sin lugar a la validación -o no- posterior, ni siquiera a la posibilidad de reflexión en el tiempo. Los docentes actuales tienen que dejar de enseñar mero conocimiento, traspasar información y datos, para enseñar algo único. Esto único es lo que hará que entonces sean las máquinas las que nunca alcancen la vara de la humanidad. Sólo cambiando la educación, los niños del futuro podrán dejar de competir con las máquinas del presente.

Comprender la naturaleza cambiante del nuevo esquema geopolítico y los nuevos modos de trabajo, va a requerir una mejor comprensión de la tecnología. Es esencial entender el lugar que ocupan determinadas tecnologías, para ser usuarios poderosos de aquellas.

Porque en todo caso, si la máquina realiza determinadas tareas de una mejor manera, ¿cuál sería el sentido de ir a la carrera por sobrepasarla? El objetivo del nuevo esquema multipolar, no es ni más ni menos que, cambiar el diálogo social. Desarrollar el mundo a partir del empoderamiento de las economías locales. Replantear lo artesanal, darle voz a los desplazados. Y no hacer esto en un tópico en particular, sino, derramar estos esfuerzos en todos los órdenes de la vida. 
Eso único que debiera enseñarse son habilidades blandas, inteligencia emocional: valores, creencias, pensamiento independiente, trabajo en equipo, características de liderazgo, saber cuidar, conocer cómo convivir con la naturaleza, tener la capacidad de compartir, etc. La práctica de deportes, la música, la plástica, y todas las artes que garanticen la enseñanza de algo diferente, y la construcción de personalidades diferentes una de la otra, contribuirán a la formación de profesionales con un desarrollo pleno, para convertirse en personas autónomas, creativas y completas, que puedan construir conocimiento, comunicarse, participar e identificar su impacto para que sean autónomos y contribuyan al entendimiento del mundo en general.

El mundo afronta el final de un sistema altamente engañoso, un período de privatizaciones y explotación. El mercado está cambiando, los trabajos están cambiando, la sociedad está cambiando, la tecnología cambia de manera tan veloz, que nadie garantiza que lo que se aprenda hoy sea útil mañana. Uno debe estar armado, preparado para afrontar eso y por tanto, generaciones actuales debieran aprender habilidades que les permitan modificar esas habilidades en un futuro. El estudio de las ciencias, las matemáticas, la reflexión histórica, la computación, el big data, son importantes, pero el pensamiento crítico, el pensamiento analítico, la comunicación y el trabajo en equipo son fundamentales para conseguir empleo, independientemente del área de desarrollo final.

Como educadores, es necesario asegurarse que los futuros egresados sean ciudadanos capaces de enfrentar los desafíos de ese mundo transformador, como aprendices constantes y no paralizarse ante ellos. No tener miedo del poder engañoso, deshonesto y brutal del poder, es la verdadera libertad.

Es obligación de cada uno reconocer que recobrar el trabajo con las manos y la mente, de manera interconectada, es la evolución más inteligente de la humanidad. Crear comunidades de trabajo y consumo, versus productores y consumidores atomizados, es la verdadera libertad.

Mirar hacia adentro para despertar, en vez de soñar mirando hacia fuera, es el único modo que existe de desarrollo en este mundo en guerra, sumido en una puja constante de intereses, fragmentado y hambriento de significado y sentido.

\section{Listado de referencias bibliográficas}

Arroyo Madera, M.A., et al. (2015). La globalización y la integración económica ¿el fin a las guerras mundiales? Recuperado el 15/12/17. Disponible en: http://revistas.unisucre.edu. co/index.php/rpg/article/view/156/198.

Asma, S. T. (2017). We could all do with learning how to improvise a little better. Recuperado el 10/12/17. Disponible en: https://aeon.co/ideas/we-could-all-do-with-learning-howto-improvise-a-little-better.

Berman, M. (1992). Todo lo que es sólido se deshace en el aire. San Pablo: Compañía de las Letras. Borges, A. (2010), en Cambariere, L. (2010). El museo de la inclusión. Sumplemento m2, Página 12, 17 de abril.

Buey Fernández, M. (2017). Involúcrame y entenderé. El gran reto de no estandarizar, sino, subir el estándard. Buenos Aires: Universidad de Palermo. 
Cambariere, L. (2017). El alma de los objetos. Buenos Aires: Paidós.

Dabbah, S. L. (2003). Globalización económica. Méjico: Ediciones Fiscales ISEF en La globalización y la integración económica, 3 de Febrero de 2015. Recuperado el: 10/11/2017. Disponible en: http://revistas.unisucre.edu.co/index.php/rpg/article/view/156/198

Dugin, A. (2018). Main factors in the development of global processes: results and prognoses. Recuperado el: 18/1/2018. Disponible en: https://www.geopolitica.ru/en/article/ globalisation-and-its-enemies.

Evans, M. (2007). Predicciones para el diseño a futuro. Revista iF. Buenos Aires: Centro Metropolitano de Diseño. Número 3 Abril. Págs: 75-80.

Flores, M. (2017). Multipolaridad. El nuevo fenómeno mundial que reemplazaría a la globalización. Recuperado el 12/11/17. Disponible en: http://www.emol.com/noticias/ Economia/2017/01/19/840649/Multipolaridad-El-nuevo-fenomeno-mundial-quereemplazaria-a-la-globalizacion.html.

Galán, B. (2006), en Cambariere L. (2006). Para dar diseño. Sumplemento m2, Página 12, 17 de abril.

Harari, Y.N. (2014). De animales a dioses. Breve historia de la humanidad. Buenos Aires, Argentina. Editorial: Penguin Random House.

Harari, Y.N. (2016). Homo Deus. Breve historia del mañana. Buenos Aires, Argentina. Editorial: Penguin Random House.

Jung, C. G. (1964). Acercamiento al Inconcsciente. En El hombre y sus símbolos. Barcelona: Paidós ISBN: 84-493-0161-0.

Ma, J. (2018). World Economic Forum. (2018) Recuperado el 25/1/18. Disponible en: https:// www.weforum.org/events/world-economic-forum-annual-meeting-2018

Naranjo, E. (2006), en Cambariere L. (2006). Para dar diseño. Sumplemento m2, Página 12,17 de abril.

Navarro, J. (2017). Mundo Multipolary Bipolar. Recuperado el: 11/12/2017 de: https://www. definicionabc.com/politica/mundo-multipolar-bipolar.php.

Néstor Canclini (2010), en Cambariere, L. (2010). El museo de la inclusión. Sumplemento m2, Página 12, 17 de abril.

Santos, M. (1993). Aceleración Contemporánea: tiempo mundo y espacio mundo. En: Fines de Siglo y Globalización. San Pablo, Hucitec-ANPUR, 1993. Recuperado el 20/12/17. Disponible en: http://www.charlespennaforte.pro.br/multipolaridad_y_globalizacion.htm.

\begin{abstract}
In a changing and globalized world, which shouts for innovation, identity and meaning, it seems that those who expect the world to adjust to its calculations and predictions are destined to be frustrated. Historically, changes have been scary because the unknown scares. However, life today is intrinsically changing, moving, disappointing and surprisingly positive. In this sense, living a life of inflexible expectations is a recipe for disaster. (Asthma, 2017) In short, the rules and structures are not divine creations. What is to be done when recognized and familiar structures fall?
\end{abstract}


The world is immersed in times of drastic changes. The powers are weaken, the masses are scattered and the earth cries. But humanity is no longer afraid of change, it seeks change; the rules follower is frustrated and the improviser adapts.

Keywords: sociology - globalization - culture - debate - design - innovation - present.

Resumo: Num mundo em mudança e globalizado, que clama por inovação, identidade e significado, parece que aqueles que esperam que o mundo se ajuste aos seus cálculos e as previsões estão fadadas a serem frustradas. Historicamente, as mudanças medo porque o desconhecido assusta. No entanto, a vida hoje é intrinsecamente mostrada, comovente, decepcionante e surpreendentemente positivo. Neste sentido, viver uma vida de expectativas inflexíveis é uma receita para o desastre (Asma, 2017). Em suma, as regras e estruturas não são criações divinas, o que você faz quando as estruturas caem onde você viveu uma vida inteira? O mundo está hoje imerso em tempos de mudanças drásticas. Os poderes são enfraquecer, as massas se dispersam e a terra chora. Mas a humanidade não tem mais medo de mudar, procure a mudança; o seguidor de regras é frustrado e o improvisador se adapta.

Palavras chave: Sociologia - globalização - cultural - debate - design - inovação - notícias.

[Las traducciones de los abstracts fueron supervisadas por el autor de cada artículo] 